\title{
Essai d'amélioration du test à la résazurine pour le contrôle de la qualité bactériologique du lait
}

\author{
par \\ M. FEUILLAT, Ch. PERROT et Ch. VIEUDRIN \\ Laboratoire de Chimie et de Technologie Agricole \\ E.N.I.T.A. de Dijon-Quétigny
}

Dans un travail effectué avec le concours de l'Ecole Nationale d'Industrie Laitière de Poligny et avec l'aide de l'Institut Technique du Gruyère, deux d'entre nous [1] ont repris en la simplifiant la méthode de Moyer et Campbell [2] qui consiste à mesurer les variations de couleur de la résazurine par photométrie d'absorption.

Cette méthode, qui permet de lire et de chiffrer avec précision les couleurs, présente en outre l'avantage, grâce à l'utilisation d'un témoin, de mesurer le seul pouvoir réducteur du lait d'origine bactériologique, à l'exclusion du pouvoir réducteur d'origine physico-chimique ou cytologique.

Appliquée d'abord à des cultures sur lait stérile de ferments lactiques ou de germes contaminants du type coliforme, puis à des laits crus ensemencés, la méthode a permis d'apporter un certain nombre de précisions sur les propriétés réductrices de ces bactéries et sur l'influence des conditions de milieu telles que la préincubation du lait.

Quelques essais ont été également tentés pour exploiter la méthode à l'échelon industriel dans le cadre du contrôle du lait en fromagerie de gruyère.

\section{A. - Méthode de lecture de la résazurine par photométrie d'absorption}

Le lait étant naturellement un milieu opaque, il est impossible d'y effectuer une colorimétrie directe de la résazurine avant ou en cours de réduction. Celle-ci doit être extraite au préalable par un solvant organique et c'est ce milieu qui est soumis à la photométrie. 


\section{I. - EXTRACTION}

Dans un tube à essai stérile, réchauffé à $37-38^{\circ} \mathrm{C}$, on mélange $5 \mathrm{ml}$ de lait à analyser avec $0,5 \mathrm{ml}$ d'une solution étalonnée de résazurine à $50 \mathrm{mg}$ de matière active par litre. L'ensemble est mis à incuber au bain-marie à l'abri de la lumière et à une température de $37-38^{\circ} \mathrm{C}$.

Après le temps d'incubation désiré : 15, 30, 45, 60 ou $90 \mathrm{mn}$, on sature le lait en bicarbonate de sodium et on ajoute $9 \mathrm{ml}$ de butanol normal, lui-même saturé en bicarbonate.

L'extraction est réalisée par 3 retournements du tube sur luimême et par centrifugation à 3000 tours par mn pendant $3 \mathrm{mn}$.

L'emploi du bicarbonate permet :

- D'annuler les effets du $\mathrm{pH}$ sur le spectre de la résazurine dans le butanol normal.

- D'augmenter sensiblement la densité optique au maximum d'absorption sans modifier la forme du spectre.

\section{II. - MESURE PHOTOMETRIQUE}

L'appareil utilisé est un spectrophotomètre Jean et Constant qui permet de faire des mesures à toutes les longueurs d'onde de la partie visible du spectre, soit de $375 \mathrm{~nm}$ à $800 \mathrm{~nm}$.

Le milieu à étudier est une solution dans le butanol normal d'un mélange de résazurine (forme oxydée, de couleur bleue) et de résorufine (forme réduite, de couleur rose).

Le graphique 1, qui donne les spectres d'absorption de la résazurine et de la résorufine dans le butanol normal, montre que le

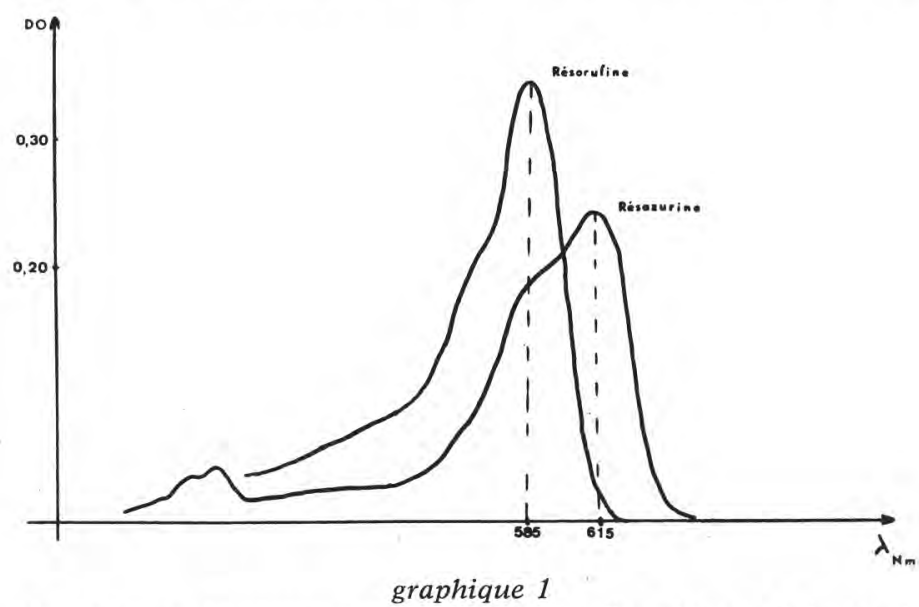

Spectres de la résazurine et de la résorufine dans le N-butanol 
maximum d'absorption se situe à $615 \mathrm{~nm}$ pour la première, et à $585 \mathrm{~nm}$ pour la seconde. En effectuant des mesures à ces deux longueurs d'onde, on obtient des valeurs de densité optique qui sont directement proportionnelles aux quantités de résazurine et de résorufine présentes dans le milieu.

Notons que par son principe (loi de Beer-Lambert), la spectrophotométrie permet d'ajuster très exactement à la même concentration toutes les solutions aqueuses de résazurine avant leur emploi. Il suffit pour cela d'établir une courbe étalon semblable à celle qui est représentée sur le graphique 2, et qui donne la proportionnalité existant entre la densité optique mesurée à $600 \mathrm{~nm}$ (maximum d'absorption de la résazurine dans l'eau), et la concentration en matière active de la solution.

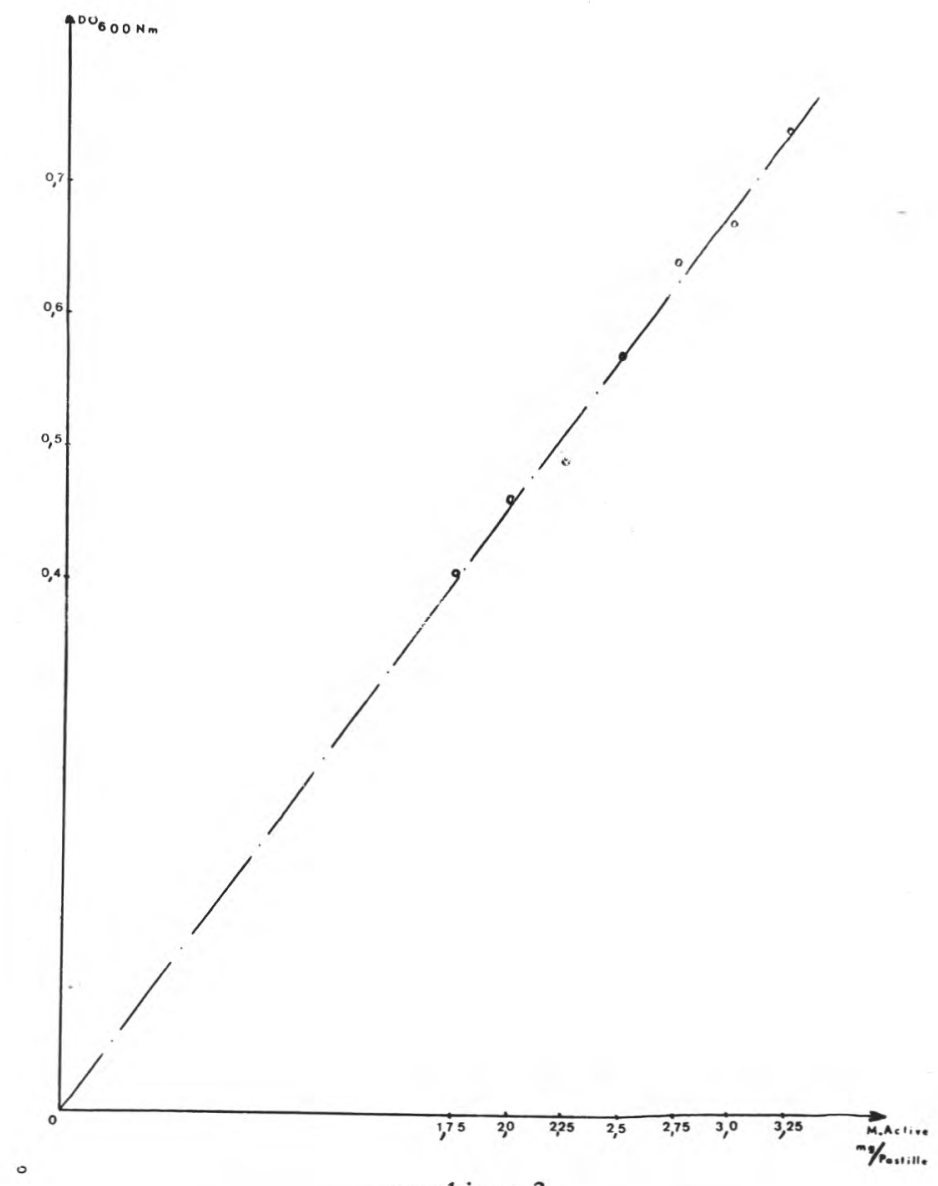

graphique 2

Ajustement des solutions de résazurine 
Cet étalonnage des solutions de résazurine à l'aide d'un spectrophotomètre ou d'un simple colorimètre, serait une pratique souhaitable dans les contrôles industriels. Nous avons pu constater en effet, que la résazurine du commerce présentée sous la forme de pastilles dosées à dissoudre dans $50 \mathrm{ml}$ d'eau, donnait des solutions de densité optique variable (de 0,40 à 0,60 ) et il s'ensuit inévitablement des erreurs lorsque l'on cherche à comparer des notes résazurine obtenues à partir de solutions différentes.

\section{III. - EXPRESSION DES RESULTATS}

L'activité réductrice des bactéries contenues dans le lait peutêtre exprimée en densité optique de différentes façons :

- Soit par la densité optique mesurée à $615 \mathrm{~nm}$ (Do 615), dont les variations correspondent à la disparition de la résazurine.

- Soit par la densité optique mesurée à $585 \mathrm{~nm}$ (Do 585), dont l'augmentation correspond à l'apparition de la résorufine.

\section{Do 615 Do 585 \\ - Soit par les rapports $\frac{}{\text { Do } 585}$ ou $\overline{\text { Do } 615}$}

- Soit encore par les différences (Do615-Do585) ou (Do585-DO615).

C'est ce dernier mode d'expression (Dosss-Do615) qui en valeur numérique nous est apparu comme le plus facile à utiliser et que nous avons retenu.

Toutes les valeurs obtenues sont corrigées par rapport à un témoin qui correspond à un lait de même origine que l'échantillon testé, mais qui, non ensemencé avec les bactéries étudiées, représente le pouvoir réducteur d'origine physico-chimique et éventuellement (cas des laits crus) l'activité réductrice de la flore naturelle.

En définitive, dans les résultats présentés ci-après, les notes spectrophotométriques indiquées correspondent à des valeurs du type :

$$
\begin{aligned}
& X=\left[\left(\text { Do585 }_{58}-\text { DO615 }_{615}\right)-\left(\text { DO585 }_{58}-D_{0615}\right)\right] \times 100 \\
& \text { échantillon témoin }
\end{aligned}
$$

et pour les différentes espèces de bactéries étudiées, nous avons établi la représentation graphique des variations de $\mathrm{X}$ en fonction du nombre de germes par ml.

Précisons que cette expression de $\mathrm{X}$ va atteindre un maximum, puis va décroître lorsqu'une partie de la résorufine se transforme en déhydrorésorufine qui est incolore et qui n'absorbe donc plus à $585 \mathrm{~nm}$. Autrement dit, la note $\mathrm{X}$ perd de sa signification pour des laits très fortement ensemencés ou pour des durées d'incubation très longues, mais nous verrons qu'elle permet cependant d'apprécier, dans les différentes espèces de bactéries, de très grandes variations de population. 


\section{B. - Protocole des essais}

\section{I. - CONTROLE DU POUVOIR REDUCTEUR DE DIFFERENTES ESPECES DE BACTERIES LACTIQUES UTILISEES COMME LEVAINS}

ET DE GERMES CONTAMINANTS DU TYPE COLIFORMES

Ce contrôle a été effectué successivement sur du lait stérile et sur lait cru.

1) Contrôle SUR LAIT STÉRILE

$1^{\circ}$ Origine et description des espèces étudiées

Il convient de se reporter au tableau 1.

En ce qui concerne les bactéries lactiques, l'étude porte sur des mélanges utilisés comme levains en fromagerie de gruyère, et non sur des espèces pures.

En ce qui concerne les coliformes, les espèces n'ont pas été identifiées. Toutefois, nous pouvons préciser que le test de Mackenzie, effectué pour le genre Escherichia, a montré qu'il ne s'agissait pas d'Escherichia coli.

\section{Technique d'ensemencement}

- Activité des souches : les espèces ou mélanges d'espèces, sont testés en phase de croissance active :

- début de l'acidification pour les bactéries lactiques,

- production de gaz pour les coliformes.

- Dilution et comptage.

Trente à quarante tubes de lait stérile sont ensemencés à des taux allant de 0 à environ $10^{7}$ germes en activité par ml, puis soumis au test de la résazurine avec lecture photométrique.

Parallèlement à ces ensemencements, des comptages sont faits sur milieux gélosés en boîtes de Pétri.

2) Contrôle SUR LAIT CRU

Les espèces ensemencées et les techniques de culture sont les mêmes que celles des essais sur lait stérile.

Le lait cru utilisé était un lait de mélange de trois vaches ayant les caractéristiques suivantes au moment de la traite :

- Flore totale acrobie : inférieure à 50000 germes $/ \mathrm{ml}$.

- Taux de coliformes : inférieur à $100 / \mathrm{ml}$.

Après ensemencement avec les différents mélanges de bactéries, deux séries de mesure à la résazurine ont été conduites :

- Les unes après préincubation à $13^{\circ} \mathrm{C}$ pendant $18 \mathrm{~h}$.

- Les autres sans préincubation. 
TABLEAU 1

\begin{tabular}{|c|c|c|c|}
\hline Dénomination & Origine & Composition & Utilisation \\
\hline $\begin{array}{l}\text { Ferments lactiques } \\
\text { Mésophiles }\end{array}$ & $\begin{array}{l}\text { Station de recherche } \\
\text { Laitière de Poligny }\end{array}$ & $\begin{array}{l}1 \text { ou } 2 \text { souches de } S \text {. Cremoris } \\
1 \text { ou } 2 \text { souches de } S \text {. Lactis } \\
1 \text { ou } 2 \text { souches de } S \text {. Diacetilactis }\end{array}$ & $\begin{array}{l}\text { Levain mésophile pour } \\
\text { la fabrication du gruyère } \\
\text { et des pâtes molles }\end{array}$ \\
\hline $\begin{array}{l}\text { Ferments lactiques } \\
\text { Thermophiles }\end{array}$ & Idem & $\begin{array}{l}1 \text { souche de L. Helveticus } \\
1 \text { souche de } S . \text { Thermophilus }\end{array}$ & $\begin{array}{l}\text { Levain thermophile pour } \\
\text { fabrication du gruyère }\end{array}$ \\
\hline Coliformes & $\begin{array}{l}\text { Isolés sur milieu au } \\
\text { désoxycholate puis } \\
\text { purifiés sur milieu } \\
\text { E.M.B. }\end{array}$ & $\begin{array}{l}1 \text { souche du genre Escherichia } \\
1 \text { souche du genre Klebsiella }\end{array}$ & \\
\hline
\end{tabular}




\section{II. - CONTROLE INDUSTRIEL DES LAITS A GRUYERE}

Cet essai porte sur 32 échantillons de laits appartenant aux producteurs d'une même fromagerie.

La répartition de ces échantillons en fonction de leur ensemencement est la suivante :

15,5 p. $100 \leqslant 100000$ germes $/ \mathrm{ml}$

$21,8 \mathrm{p} .100 \leqslant 200000$ germes $/ \mathrm{ml}$

25 p. $100 \leqslant 300000$ germes $/ \mathrm{ml}$

60 p. $100 \leqslant 500000$ germes $/ \mathrm{ml}$

En prenant comme méthode de référence la méthode directe de numération microbienne (ou méthode Thomson), nous avons cherché à comparer le test classique à la résazurine et la méthode avec lecture spectrophotométrique en vue de classer les laits selon leur qualité bactériologique.

Précisons que dans les résultats présentés ci-dessous, le test classique à la résazurine a été effectué dès la réception du lait à la fromagerie, alors que la méthode avec lecture photométrique a été appliquée à des échantillons ayant séjourné en glacière à 6-7 $\mathrm{C}$ pendant $18 \mathrm{~h}$.

\section{C. - Résultats}

Dans les deux séries d'essais précédemment définies, notre but était de savoir si le test à la résazurine amélioré par une lecture spectrophotométrique permettait :

- D'une part, d'établir une corrélation entre le pouvoir réducteur des bactéries et leur taux d'ensemencement; l'intérêt étant une utilisation possible de la méthode pour le contrôle de l'activité des levains ou le contrôle de la maturation des laits en fromagerie.

- D'autre part, de classer des laits à l'échelon industriel selon leur qualité bactériologique.

\section{I. - CORRELATION ENTRE LE POUVOIR REDUCTEUR ET LE TAUX D'ENSEMENCEMENT DES BACTERIES}

\section{1) Présentation des résultats}

Les résultats sont donnés, comme nous l'avons indiqué plus haut, sous la forme de graphiques établissant les variations de la note résazurine $\mathrm{X}$ en fonction du nombre de germes par $\mathrm{ml}$.

Etant donné la gamme étendue des populations testées, nous retenons, non pas le nombre $\mathrm{N}$ de germes inoculés par $\mathrm{ml}$, mais son logarithme. Par ailleurs, pour limiter les erreurs dues à la technique des dilutions et des comptages sur milieux gélosés, nous regroupons les populations en classes équivalentes et pour chaque classe, nous faisons la moyenne des notes $\mathrm{X}$ obtenues à la résazurine, soit $\overline{\mathrm{X}}$. 


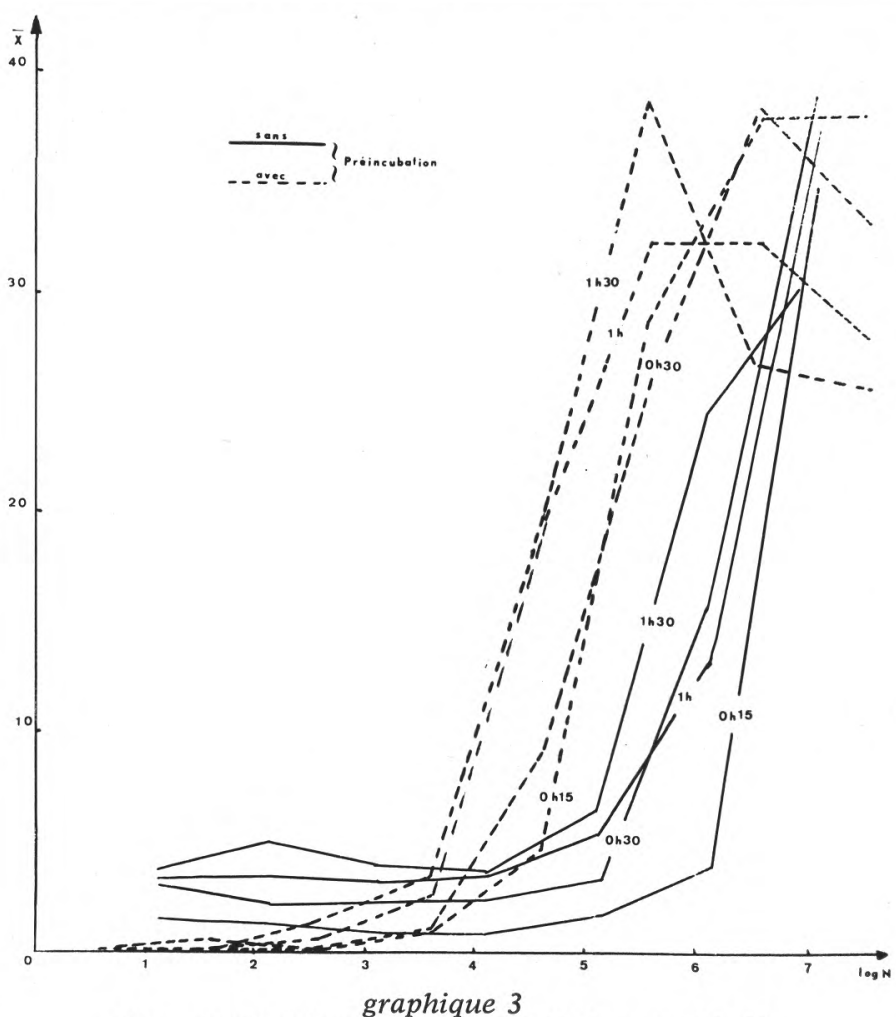

Ferments lactiques mésophiles (lait cru $-37^{\circ} \mathrm{C}$ )

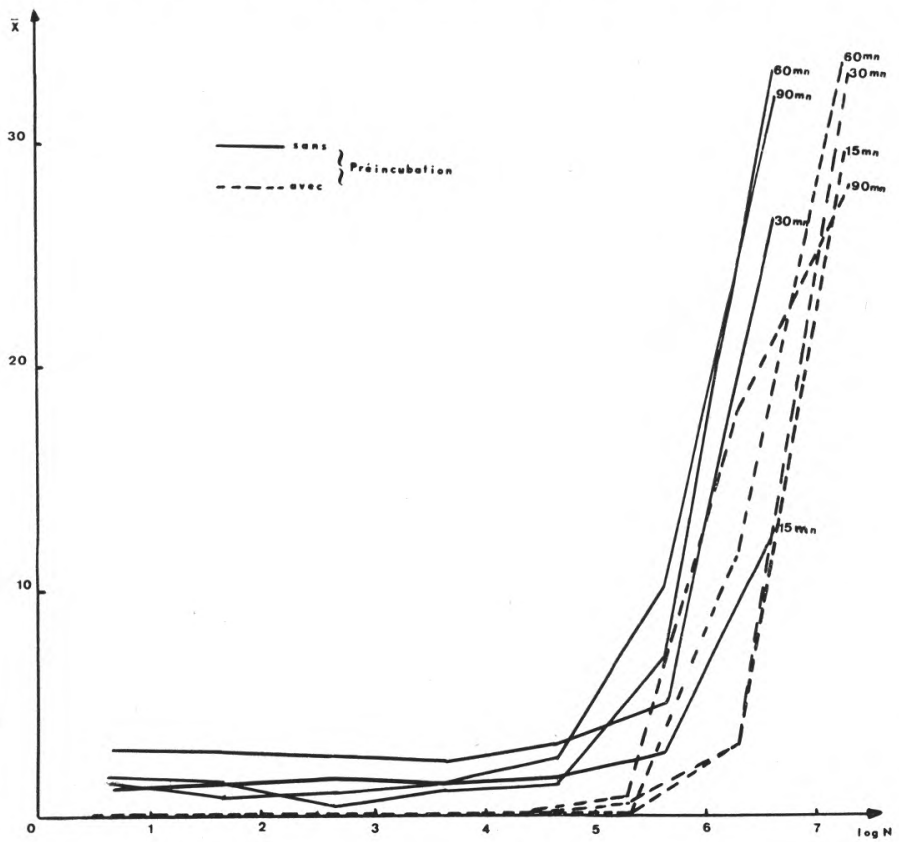

Graphique 4

Ferments lactiques thermophiles (lait $\mathrm{cru}-37^{\circ} \mathrm{C}$ ) 
De nombreux graphiques ont été ainsi tracés. Nous n'en reproduisons ici que quelques-uns : graphiques 3,4 et 5 , qui se rapportent chacun à une catégorie de germes testés sur lait cru avec ou sans préincubation.

A chaque courbe, c'est-à-dire à un temps d'incubation donné, correspond un taux d'ensemencement minimum à partir duquel la réduction de la résazurine devient significative. Nous appellerons cette valeur particulière de population "seuil de sensibilité ».

Le seuil de sensibilité est exprimé par le nombre de germes par ml qui correspond à la limite inférieure de la classe de population dans laquelle il se situe.

Le tableau 2 regroupe les valeurs des seuils de sensibilité obtenues pour les différentes variantes du protocole d'essais.

\section{2) Discussion}

Les résultats du tableau 1 montrent que la sensibilité de la méthode à la résazurine pour apprécier le taux d'ensemencement d'un lait est très valable selon la nature de la flore inoculée, mais selon aussi un certain nombre d'autres facteurs : ensemencement

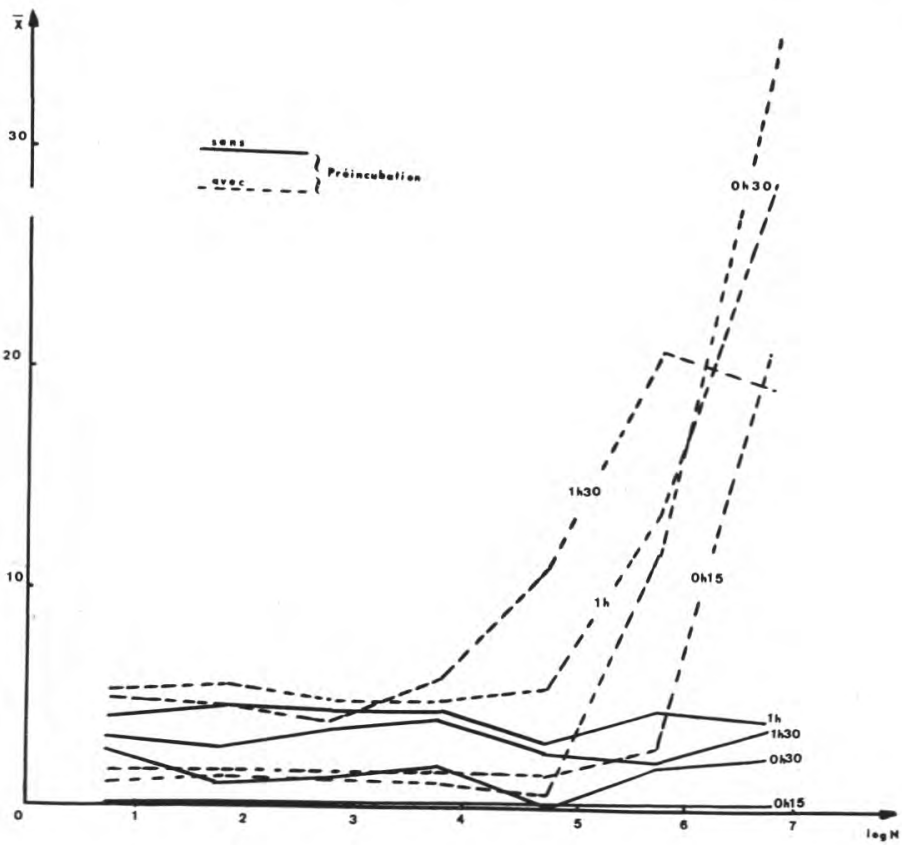

graphique 5

Coliforme du genre Klebsiella (lait cru $-37^{\circ} \mathrm{C}$ ) 
TABLEAU 2. - Tableau des seuils de sensibilité

\begin{tabular}{|c|c|c|c|c|c|}
\hline Espèce inoculée & Substrat Durée d'incubation & $0 \mathrm{~h} 15$ & $0 \mathrm{~h} 30$ & $1 \mathrm{~h}$ & $1 \mathrm{~h} 30$ \\
\hline Ferments lactiques mésophiles & $\begin{array}{l}\text { Lait stérile } \\
\text { Lait cru avec préincubation } \\
\text { Lait cru sans préincubation }\end{array}$ & $\begin{array}{r}37600 \\
3030 \\
1360000\end{array}$ & $\begin{array}{r}37600 \\
3030 \\
136000\end{array}$ & $\begin{array}{r}2590 \\
3030 \\
136000\end{array}$ & $\begin{array}{r}2590 \\
3030 \\
136000\end{array}$ \\
\hline Ferments lactiques thermophiles & $\begin{array}{l}\text { Lait stérile } \\
\text { Lait cru avec préincubation } \\
\text { Lait cru sans préincubation }\end{array}$ & $\begin{array}{r}629500 \\
3370000 \\
460000\end{array}$ & $\begin{array}{r}629500 \\
3370000 \\
460000\end{array}$ & $\begin{array}{r}55750 \\
337000 \\
46000\end{array}$ & $\begin{array}{r}55750 \\
337000 \\
46000\end{array}$ \\
\hline Coliformes du genre Escherichia & $\begin{array}{l}\text { Lait stérile } \\
\text { Lait cru avec préincubation } \\
\text { Lait cru sans préincubation }\end{array}$ & $\overline{-}$ & 490000 & $49 \overline{000}$ & $4 \overline{900}$ \\
\hline Coliformes du genre Klebsiella & $\begin{array}{l}\text { Lait stérile } \\
\text { Lait cru avec préincubation } \\
\text { Lait cru sans préincubation }\end{array}$ & 560000 & $56 \overline{000}$ & $\begin{array}{r}1210000 \\
56000\end{array}$ & $\begin{array}{r}1210000 \\
5600 \\
-\end{array}$ \\
\hline
\end{tabular}


sur lait stérile ou sur lait cru, ensemencement sur lait cru suivi ou non d'une préincubation.

\section{$1^{\circ}$ Influence de la nature flore}

Les essais sur lait stérile sont les plus rigoureux pour essayer de classer les différentes catégories de germes testés selon leur pouvoir réducteur.

D'après les valeurs des seuils de sensibilité obtenues dans ces essais, on constate que les ferments lactiques mésophiles sont beaucoup plus réducteurs que les ferments lactiques thermophiles et que la réduction de la résazurine par les coliformes est négligeable, si ce n'est pour des populations anormalement fortes : plus de $10^{\circ}$ germes par ml pour le genre Klebsiella.

Nous donnons ci-dessous les notes moyennes obtenues à la résazurine pour les différentes bactéries avec un ensemencement de $10^{6}$ germes par $\mathrm{ml}$ et une durée d'incubation à $37^{\circ} \mathrm{C}$ de $15 \mathrm{mn}$ :

Ferments lactiques mésophiles $\quad x=16$

Ferments lactiques thermophiles $x=4$

Germes coliformes $\quad x=0$

$2^{\circ}$ Influence de la nature du substrat : Ensemencement sur lait stérile ou sur lait cru

Pour l'étude de ce facteur, il y a lieu de comparer les essais conduits sur lait stérile et ceux effectués sur lait cru sans préincubation.

Dans ces derniers, nous pouvons relever des seuils de sensibilité nettement plus élevés pour les ferments lactiques mésophiles. Ceux-ci semblent très sensibles à la concurrence de la flore indigène du lait, à l'inverse des ferments thermophiles dont les seuils de sensibilité sont au contraire abaissés.

Comme sur lait stérile, la résazurine ne permet pas sur lait cru sans préincubation de déceler la présence des coliformes, dont les seuils de sensibilité demeurent indéterminés.

\section{3o Influence de la préincubation du lait cru}

Une préincubation de $18 \mathrm{~h}$ à $13^{\circ} \mathrm{C}$, améliore considérablement la sensibilité de la méthode à la résazurine en ce qui concerne la mesure de l'activité des ferments lactiques mésophiles. Celle-ci peut alors être décelée pour de faibles taux d'ensemencement : de l'ordre de 3000 germes par $\mathrm{ml}$, et après $15 \mathrm{mn}$ seulement d'incubation à $37^{\circ} \mathrm{C}$.

Par contre, la même préincubation semble gêner le développement des thermophiles dont les seuils de sensibilité sont alors très élevés : 10 fois supérieurs à ceux obtenus sans préincubation.

En ce qui concerne les coliformes, leur mise en évidence par la résazurine devient possible pour une population initiale voisine de 5000 germes par ml après une incubation à $37^{\circ} \mathrm{C}$ de $1 \mathrm{~h} 30$. 


\section{II. - CLASSEMENT INDUSTRIEL DES LAITS DESTINES A LA FROMAGERIE DE GRUYERE}

Dans cet essai, rappelons-le, nous avons cherché à comparer le test classique à la résazurine à la méthode suivie d'une lecture photométrique pour classer les laits selon leur qualité bactériologique ; la méthode de référence étant la méthode directe de numération microbienne.

Nous nous sommes limités à faire deux catégories de laits avec des seuils de population choisis arbitrairement de la façon suivante :

Plus ou moins 100000 germes $/ \mathrm{ml}$

Plus ou moins 200000 germes $/ \mathrm{ml}$

Plus ou moins 500000 germes $/ \mathrm{ml}$

Dans les tests à la résazurine, nous avons retenu comme limites entre les deux classes de laits (ou seuils de réduction) :

- La note 4 après $1 \mathrm{~h} 30$ d'incubation à $37^{\circ} \mathrm{C}$ dans le test classique.

- La note 7,5 après $30 \mathrm{mn}$ d'incubation à $37^{\circ} \mathrm{C}$ dans la méthode photométrique.

Le tableau 3 montre que la lecture photométrique, avec une incubation de $30 \mathrm{mn}$ seulement, permet, dans tous les cas, un classement des laits beaucoup plus proche de celui obtenu par numération de la flore totale, que l'appréciation visuelle.

81 p. 100 des laits étudiés ont pu ainsi être classés de la même façon lorsque l'on retient 100000 germes $/ \mathrm{ml}$ comme seuil de discrimination entre laits de bonne et laits de mauvaise qualité bactériologique.

TABLEAU 3

Pourcentage de laits classés de la même façon que par la numération microbienne

\begin{tabular}{|c|c|c|c|}
\hline $\begin{array}{l}\begin{array}{l}\text { Seuil } \\
\text { réduction }\end{array} \\
\begin{array}{r}\text { Seuil de population } \\
(\text { germes } / \mathrm{m})\end{array}\end{array}$ & 100000 & 200000 & 500000 \\
\hline $\begin{array}{l}\text { Note } 4 \\
\text { (Notation visuelle de la résazurine } \\
\text { après } 1 \mathrm{~h} 30 \text { d'incubation) }\end{array}$ & 40,6 p. 100 & 59,0 p. 100 & 53,0 p. 100 \\
\hline $\begin{array}{l}\text { Note } 7,5 \\
\text { (Notation photométrique de la résa- } \\
\text { zurine après } 30 \mathrm{mn} \text { d'incubation) }\end{array}$ & 81,2 p. 100 & 75,0 p. 100 & 69,0 p. 100 \\
\hline
\end{tabular}




\section{Conclusion}

Alors que le contrôle bactériologique du lait revêt une grande importance en vue du paiement à la qualité, il nous est apparu utile de rechercher si une méthode classique telle que le test à la résazurine, encore très pratiquée dans la région du gruyère à cause de sa simplicité et de sa rapidité, pouvait être améliorée.

La préférence donnée à la résazurine plutôt qu'au bleu de méthylène comme indicateur d'oxydo-réduction se justifie lorsque l'on est dans l'obligation d'avoir très rapidement des renseignements sur l'ensemencement d'un lait. C'est le cas en fromagerie de gruyère où une partie du lait est mise en fabrication dès son arrivée à la fruitière.

La première idée qui a présidé à ce travail a été de substituer à l'œil un spectrophotomètre pour la lecture des différentes teintes prises par la résazurine en cours de réduction, et qui, dans la méthode classique, fait l'objet d'une notation conventionnelle de 6 à 0 toujours imprécise. L'œil, en effet, ne peut apprécier avec précision toutes les nuances de couleur, ni les comparer entre elles. Mesurées au spectrophotomètre, ces nuances sont traduites en valeurs chiffrées de densités optiques.

Par ailleurs, l'utilisation d'un spectrophotomètre ou d'un colorimètre permet l'étalonnage des solutions de résazurine avant leur emploi, et les possibilités de correction par rapport à un témoin sont d'un grand intérêt, en particulier pour mesurer d'une façon spécifique l'activité d'une flore sélectionnée sous la forme d'un levain.

La corrélation qui existe entre la réduction de la résazurine par un lait et son taux d'ensemencement, dépend de la qualité de la flore. Il existe pour chaque espèce un seuil de population au-dessous duquel la réduction de la résazurine n'est pas significative.

Les valeurs des seuils de sensibilité obtenues pour les ferments lactiques mésophiles : 3000 germes par ml dans du lait préincubé à $13^{\circ} \mathrm{C}$ pendant $18 \mathrm{~h}$, environ 150000 germes par ml dans du lait non préincubé, permettent d'envisager une utilisation de la méthode à la résazurine pour mesurer l'activité de ces ferments dans le contrôle de la maturation des laits ou dans le contrôle des levains de fromagerie avant et pendant la fabrication. Nous poursuivons actuellement des essais dans ces deux domaines.

En ce qui concerne le classement industriel des laits selon leur qualité bactériologique, le nombre d'essais réalisés n'est pas suffisant pour apprécier la valeur réelle de la méthode. Il n'en reste pas moins vrai que la lecture de la résazurine par photométrie devrait permettre un classement beaucoup plus proche de celui obtenu par les méthodes de numération microbienne que le test classique. 


\section{S u m m a ry}

An attempt to improve the resazurin test

for estimating the bacteriological quality of milk

An easy method for the extraction and photometric reading of resazurin is suggested. The new possibilities offered by this methode are as follows :

- Calculation of the optical densities of the different colours that develop in the course of reduction.

- Calibration of resazurin solution before use.

- Correction of readings by means of a standard.

This methode has allowed determination of sensitivity threshold for different lactic ferments used in cheese-making and the results permit us to think of applying it to the testing of milk maturation and ferment activity.

A few experiments in industrial grading of milk according to its bacteriological quality yieldedresults much nearer to those obtained by determining bacterial numbers than the usual resazurin test.

Reçu pour publication en mars 1971.

\section{Références bibliographiques}

[1] Perrot (Ch.) et Vieudrin (Ch.) (1970). - Essai d'amélioration du test à la résazurine pour le contrôle bactériologique du lait, mémoire présenté pour l'obtention du diplôme d'Ingénieur des Techniques Agricoles, E.N.I.T.A. de Dijon-Quétigny.

[2] MoYer (R. H.) and CAMPBell (J. J. R.) (1963). - Mechanism of resazurin réduction in milk of low bactérial content. Journal Dairy Science, t. 46, $\mathrm{n}^{\circ} 9$, p. 897. 